\title{
Toponimia de Ticapampa
}

\author{
Ana Arias Torre \\ Departamento Académico de Lingüística
}

\begin{abstract}
a toponimia es un estudio de carácter social, que parte del registro de nombres de lugar que hayan recibido una o más denominaciones por parte del hombre, durante su periodo de poblamiento en grandes civilizaciones, tal inventario pasa por el análisis lingüístico de sus formas. Debido a su carácter social es considerada como un caudal de datos culturales, si nos referimos a los aportes etnolingüísticos que da luz. En la lingüistica, la toponimia desempeña un papel importante para el estudio sincrónico y diacrónico de las lenguas nativas, actuales o extintas, que se desarrollan o se han desarrollado en un territorio. Además, es una fuente de información léxica directa porque los topónimos en su forma son testigos del cambio lingüístico que opera en una determinada lengua. Así los estudios toponímicos se apoyan básicamente, en la lingüística, para hacer un análisis de las formas léxicas, identificar los procesos lingüisticos involucrados en la formación de topónimos y para dar una descripción de los elementos constituyentes en las estructuras foponímicas.
\end{abstract}

\section{"Jorge Puccinelli Converso"}

Antes de dar algunos alcances de nuestro estudio, cabe mencionar que, Ticapampa' es un pueblo de tradición minera, de geografia muy accidentada, de profundidad variable, con una vegetación silvestre, graminea, arbustiva y herbácea.

Este estudio ${ }^{2}$ se inicia con el recojo de un corpus toponímico representativo del lugar formado por ciento cincuenta y uno nombres, con el objetivo principal de describir los procesos lingüisticos operados en la formación de los nombres de lugar y; en consecuencia, confirmar la presencia de grupos lingüisticos que ocuparon esta área demográfica. Asimismo, registrar geográficamente la toponimia recogida y remarcar la importancia de la geografía lingüística y de la etnolingüística en esta clase de trabajo.

1 Es uno de los diez distritos que comprende la Provincia de Recuay en el Departamento de Ancash, con una altitud de 3000 a $4000 \mathrm{msnm}$.

2 El presente articulo presenta las conclusiones derivadas de la tesis del mismo nombre. 
Los procesos registrados en el corpus son de naturaleza fonológica ${ }^{3}$, morfológica y sintáctica. A continuación presentamos nuestras conclusiones acompañadas de algunos ejemplos representativos:

1. En el nivel fonológico, la asimilación progresiva y regresiva que experimentan las vocales altas: /i, u/ frente a un segmento postvelar o su variante fricativa sonora [ $\gamma]$, que se muestra en la transcripción castellana de los nombres quechuas, en el cuadro $l$ y 2 .

\section{CUADRo 1}

ASIMILACIÓN PROGRESIVA
(1) /pišqu wayin/ [piš $\gamma$ o wayin] (THQA)

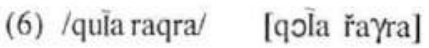
(2) /qiru quèa/ [qعro qoča]
(7) /quīpa ruri/ [qoîpa uri]
(3) /qinwayuq/ [qenuwa -yo $\chi]^{5}$
(8) /hiquš/
[he $\gamma_{0}-5$ ]
(4) /qumpayna/
[qompe: -na]
(9) /qiīay hirka/
[qॄēe: hirka]
(5) /quyIur/
[qoyĪur]
(10)/anqaq/
$[\mathrm{aN} \gamma \mathrm{a}-\chi]^{6}$

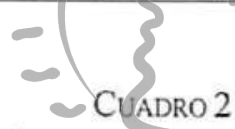
ASIMILACION REGRESIVA
(1) /puqraq/

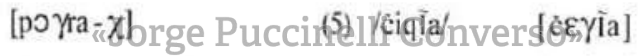
(2) Kunkas puntal [kunka-spunta] $]^{7}$ (THQC)
(6) /mašrauqu [mašraoło]
(3) /wawyanga/ [wawya-N $\gamma$ a]

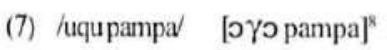
(4) /qinuwayuq [qEnuwa-yo $\chi$ ]
(8) /unqayuqruri/ [oN $\gamma$ ayo $\chi \check{r} u r i]>[0 \mathrm{~N} \gamma$ ayəo uri]

\footnotetext{
3 No presentamos las reglas que explican estos procesos para no extendernos en este aspecto, particularmente.

4 Proceso básico de todo cambio fonológico, consiste en el cambio de rasgos que experimentan fonemas cercanos.

5 Se usa el simbolo $[\chi]$ para representar a la variante posvelar fricativa sorda.

${ }^{6}$ El simbolo $[\gamma]$ representa a la variante fricativa posvelar sonora.

7 Topónimo hibrido quechua-castellano (THQC, topónimo hibrido quechua aru (THQA).

s Ocopampa, nombre anterior del pueblo joven Santa Gertrudis.
} 
Las vocales y consonantes pueden adquirir rasgos articulatorios propios de otros segmentos, en los ejemplos mostrados, las vocales altas asimilan los rasgos articulatorios de la consonante oclusiva post velar $/ \mathrm{q} / \mathrm{y}$ de sus variantes, la fricativa postvelar sorda $[\chi]$ y la fricativa postvelar sonora $[\gamma]$, que aparecen después de vocal. La abertura vocálica ocurre en dos posiciones?:

1. Cuando la vocal alta antecede $\mathrm{a} / \mathrm{q} /$.

2. Cuando la vocal alta sigue a este segmento, esta posición es tratada en la asimilación progresiva.

En (3) y (8) la consonante nasal dentoalveolar adquiere rasgos articulatorios del mismo elemento oclusivo postvelar/q/, cuando precede a este segmento. En el caso (7) y (8) registramos el cambio inicial de $u>[0]$ por influencia de $/ \mathrm{q} /$, posteriormente, el debilitamiento y pérdida de la postvelar por influencia del contexto, en final de palabra, manteniéndose vigente la vocal con timbre bajo, como huella de que existió un segmento postvelar.

Los cambios, $\mathrm{i}>\varepsilon, \mathrm{u}>0$ producidos porla presencia de /q/ se mantienen inalterables ante la presenciade sus variantes $[\gamma, \chi]$, éstas no permiten que las vocales recuperen su timbre alto. $\quad\left\{\quad \mathrm{n} />[\mathrm{N}] \quad \_/ \mathrm{q} /\right.$

Las vocales /i, u/ se realizancomo tal, conservando su timbre alto en todo ambiente no cercano a la consonante postvelar /q/, como demuestran los ejemplos. La postvelar /q Pilantienetse crasgol te botisiva sorda en todo ambiente

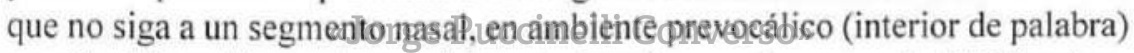
o en final de palabra, estos cambios son descritos en el proceso de debilitamiento que sufre el segmento postvelar.

El debilitamiento, con la espirantización de la oclusiva postvelar/q/, registrado en el cuadro 3; la sonorización de las oclusivas $/ \mathrm{t}, \mathrm{k} /{ }^{10}$ en el cuadro $5 \mathrm{y} / \mathrm{q} /$ en posición intervocálica, en el cuadro 4; y la aspiración de la fricativa alveolar /s/ en posición (\#), posteriormente la expansión del cambio en posición (V_V) en el cuadro 6.

Parker. en 1976, reconoce estos dos ambientes de apertura vocálica.

${ }^{10}$ Cambio inicial que se expandió en esta variedad por contacto con el castellano. 


\section{Cuadro 3}

\section{EN POSICIÓN IMPLOSIVA}

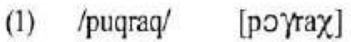
(6) /sị̣̄a/
[č́үَ̄a]
(2) lanqaq/ $[\mathrm{aN} \gamma \mathrm{a}-\chi]$
(6) Auyaq luyaq/
[luya $-\chi$ luya $-\chi$ ]
(3) /qayaq/ [qaya- $\chi]$
(7) /čuriyaq/
[c̈uriya: $\chi$ ]
(4) /qinwayuq/ [qEnwa-yo $\chi$ ]
(8) /wačaqpampa/
[wača- $\chi$ pampa]
(5) /rampaq [ampa- $\chi]$
(10) /unqayoq ruri/[ [ $\mathrm{N} \gamma \mathrm{a}-\mathrm{y} \supset \chi \mathrm{uri}]>[\mathrm{oN} \gamma \mathrm{a}-\mathrm{y} \odot \circ$ uri]

En su realización la consonante oclusiva postvelar, en posición final de palabra se fricativiza, convirtiéndose en una consonante fricativa postvelar sorda $[\chi]$ y en final de sílaba, siempre que no esté seguida de una consonante sonora (r o ll). En el cuadro 4, sólo el caso 5) muestra cómo la fricativa postvelar sorda termina desapareciendo en esta posición. Proponemos la siguiente regla:

\section{CuAnRo 4}

EN AMBIENTE VOCALICO

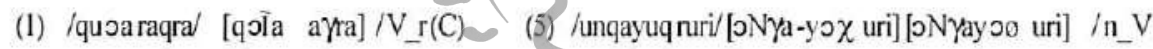

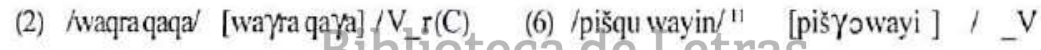

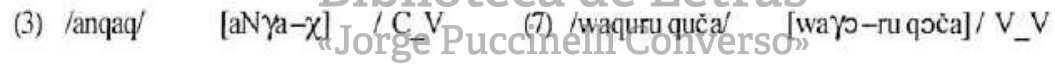
(4) /mašrauqu/ [mašraojo] /V_V
(8) $/ \bar{c} \varepsilon q \bar{I} /$
[c் $\varepsilon \gamma \bar{a} a]$
IV_II (V)

Los topónimos muestran que la postvelar sorda /q/ se convierte en una fricativa postvelar sonora $[\gamma]^{12}$, en contexto prevocálico, en interior de palabra y cuando le sigue una consonante sonora (lateral palatal, vibrante simple, o cuando sigue a una consonante nasal) o se convierte en una fricativa postvelar sorda $[\chi]$, en posición final de palabra. Gary Parker señala que este proceso es característico de las variedades de las provincias del Callejón de Huailas. Sin embargo, registramos a /q/ sonorizada tras vocal y seguida de consonantes (r o ll), a través, de estos ejemplos demostramos que en el habla de Recuay se puede encontrar la forma

"Topónimo hibrido quechua-aru (THQA).

12 Simbolo usado por Cerrón Palomino. Para el mismo simbolo Parker emplea $[\mathrm{g}]$ y $[\mathrm{x}]$ para su variante sorda. 
sonorizada de /q/. Cerrón Palomino reconoce la sonorización de la oclusiva postvelar como una vibrantización y lo explica en su Lingüistica Quechua.

CUADRO 5

SONORIZACIÓN ${ }^{13}$

$\begin{array}{lllll}\text { (1) kaša pukru } & > & \text { kaša øukru } & \\ \text { (2) učpa pukru } & \sim & \text { uợa øukru } & \\ \text { (3) kuntur wayin } & \sim & \text { kondor wayin } & \text { (THQA) } \\ \text { (4) tranka } & > & \text { tranga (TC) } & \end{array}$

Proceso influenciado mucho por el castellano, en el caso (1), la oclusiva bilabial / $\mathrm{p} /$ desaparece en posición inicial de palabra, no registrándose el paso de $p>b$. En el caso (2) parece ser un proceso reciente, en el uso la forma conservadora alterna con la forma eliminada, como en el ejemplo (2) la oclusiva bilabial desaparece en posición inicial de palabra, pero interior de frase. En (3) ocurre la sonorización de ${ }^{*} \mathrm{t}>\mathrm{d}$, en muchos casos, influenciado por el castellano. En el ejemplo (4), $/ \mathrm{k}$ / experimenta una sonorización convirtiéndose $\mathrm{a} / \mathrm{g} /$.

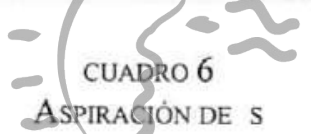

(1) Rahupunta < rasupunta (THQC)

(2) Hinčispampa Bỉblsinçis pampa de Letras

(3) Hupay

(4) Pukarahu "Jorg supay ccinelli Converso» $<$ pukarasu

(5) Kondorwayin $<$ kondorwaøin $<$ kunturwahin $<$ kunturwasin (THQA)

(6) Pišquwayin $<$ pišquwaøin < pišquwahin<pišquwasin (THQA)

La aspiración de */s/ es un cambio anterior que afectó al Quechua Central y sigue vigente en esta variedad. En los ejemplos (2) y (3), el cambio se encuentra en un estado inicial, en el que $* / \mathrm{s} />/ \mathrm{h} / / \#_{-}$, expandiéndose después en ambiente (V_V), como en los ejemplos (1), (4), (5) y (6). El cambio se profundiza en (5) y (6), provocando la caída de la / $\mathrm{h} /$ intervocálica, derivada del cambio inicial de aspiración, este proceso es observado en las provincias del Callejón de Huaylas, Bolognesi, Aija y en el sur de Huari.

13 Consiste en la adquisición del rasgo de sonoridad por parte de un fonema sordo, en contacto con in segmento sonoro: generalmente. seguido o precedido por una vocal o consonante sonora. 
La supresión ${ }^{14}$, con la elisión de $/ \mathrm{h} /$ en posición (V_V), la elisión de vocal en final de palabra y la elisión de fonema en límite morfémico.

\section{Cunado 7}

ELISIÓN DE FONEMA EN LIMITE MORFĖMICO

(1) tupuk hirka > tupuk irka

(2) sillaqa hirka $>$ sil̄agas øirka

(3) waka hirka waka oirka
(4) kaša alameda kaša olameda (THQC)

(5) ičik kurus - ičik urus

En (1) y (2) se pierde la fricativa glotal $/ \mathrm{h} /$, derivada del proceso inicial de aspiración, la fricativa alveolar/s/, en posición inicial de palabra, cuando sigue a una consonante sorda o a una vocal del primer compuesto.

El ejemplo (2) muestra la caida de la consonante fricativa glotal en posición inicial de palabra, precedida por una fricativa palatal sorda u oclusiva velar sorda final del primer compuesto, aunque muchas veces en el uso se observa la resistencia a la caida de la consonante.

En (4) y (5) se observa la caída de la vocalo consonante inicial del segundo compuesto, debido a que el primer compuesto termina en la misma vocal o consonante, con la que empieza el segundo. En la cadena hablada, se observa que la elisión de fonemas ocurre sistemáticamente cuando no están separadas por una pausa.

Biblioteca de Letras

\section{"Jorge Puccinelli Converso»}

ELISIÓN DE VOCAL

\begin{tabular}{|c|c|c|c|c|}
\hline cuyan & $<$ & cuya -no & $<$ & ${ }^{*}$ cuya - na \\
\hline kiswar pacan & $<$ & kiswar paca -no & $<$ & * kiswar paca -ni \\
\hline pišqu wayin & $<$ & pišqu wayi -no & $<$ & * pišqu wayi -ni \\
\hline kundor wayin & $<$ & kundor wayi -no & $<$ & * kuntur wayi -ni \\
\hline pacan posada & $<$ & pacanø posada & $<$ & ${ }^{*}$ paca -ni posada (THQA C) \\
\hline wacaw n tsaka & $<$ & wacaw -no tsaka & $<$ & * wacaw -ni tsaka \\
\hline rikanan hirka & $<$ & rikana-no hirka & $<$ & * rikana-ni hirka \\
\hline isku pacan & $<$ & isku paca - no & $<$ & * isku paca $-n i$ \\
\hline
\end{tabular}

${ }^{14}$ Proceso que produce la pérdida de una consonante o vocal en posición inicial de palabra. 
Consiste en la pérdida de la vocal del sufijo, en posición final de palabra, ocupando la vocal elidida una posición postónica en la palabra, por ello se observa que los nombres que presentan este supuesto morfema derivativo tienen un acento agudo y no grave, como debería ser en el quechua. Las vocales forman parte de sufijos nominalizadores como \{-na\} en (1) y del sufijo derivativo aru $\{-n i\}$ el resto de ejemplos, sufijo del que hablaremos especialmente en el análisis morfológico.

Primeramente, hemos considerado posible que el sufijo presente en los topónimos del cuadro 7 provenga del quechua, como sufijo flexivo, marcador de tercera persona, en raices nominales quechuas, a excepción del primer caso; o sean procedentes del aru, como sufijo de derivación nominal presente frecuentemente en la formación de nombres toponimicos. No se ha descartado esta última posibilidad, porque, históricamente parte del área que comprende el QI, estuvo ocupado por grupos de habla aru

La sustitución, con la alveolarización de /è/ y la monoptongación de /ay, iy, aw/.

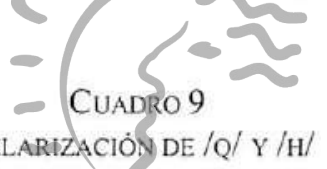

(1) raçaq pampa $>$ raçak pampa (3) qumpayna $\quad$ kompayma

(2) pačaq pampa B paok paj̣aca. (4L hireis panpa xincis pampa "Jorge Puccinelli Converso"

Es un cambio, más bien externo, registrado en la población bilingüe y castellanohablante, este proceso motiva el cambio del fonema oclusivo postvelar $y$ fricativo glotal por una oclusiva y fricativa velar sorda respectivamente. Se observa el uso variable de la forma velarizada del fonema frente a la forma conservadora, este cambio tiene nivel fonémico cuando se confunde con los fonemas del castellano. En los ejemplos (1) y (2) el cambio ha finalizado mientras que en el resto recién se inicia el cambio porque se registra una alternancia.

CUADRO 10

LA MONOPTONGACION
(1) /wayçaw isku/
[we:čo: isku]
(2) /sala mačay/
[sala mače:] [sala mačeø] (THCQ)
(3) /èawpis/
[čo:pis] 

(4) /wanka pituy/
[wanka piti:] [wanka pitiø]
(5) /purway punta/
[purwe: punta] (THQC)
(6) /hupay punta/
[hupe: punta] (THQC)
(7) /mačay kanča/
[mac̀e: kanča]
(8) /quylur/
[qoylur]

Es un cambio interno que consiste en la reducción vocálica, que experimenta un diptongo al convertirse en monoptongo, con este proceso se forman las vocales largas de abertura media. En el comportamiento vocálico de la variedad Ancash, de algunas zonas del Callejón de Huailas, se observa el proceso de monoptongación que produce un acortamiento vocálico de determinadas secuencias: ay, aw y uy. El sistema vocálico acorta estas secuencias, dándoles un rasgo adicional de cantidad, probablemente, para equilibrar la pérdida de las secuencias vocálicas y no hacer drástico el cambio, convirtiendo los diptongos en vocales medias largas (e:, o:, i:); estas vocales sufren un acortamiento variable, en posición final de frase y cuando se encuentran en sílaba trabada; esto muestra, probablemente, la tendencia del sistema a la eliminación del rasgo de cantidad en las vocales derivadas del proceso de monoptongación, el diptongo /uy/ se acorta sólo en posición final de frase, cuando sigue a un segmento posvelar. Además, con la monoptongación el sistema cubre la ausencia de vocales medias, formando cinco vocales breves y largas, demostrando que los topónimos son formas que sufren cambios constantes y no son necesariamente formas congeladas.

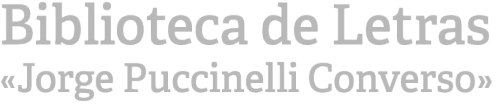

CuAdro 11

Alveolarización DE / / $/$

\begin{tabular}{lll}
\hline (1) pacu yaku & $<$ & paču yaku \\
$(2)$ caki qoča & $<$ & čaki qoča \\
(3) wačawnø caka & $<$ & wačawni čaka \\
\hline
\end{tabular}

El segmento africado palatal sufre un cambio en su estructura articulatoria, con una adelantamiento a la posición dentoalveolar. Cambio propio de esta variedad y en posición inicial de silaba y palabra.

La fusión, con la despalatalización de $/ \tilde{n} /{ }^{15}$ y $/ \check{s} /$.

:5 Cambio muy antiguo en el quechua de esta variedad. 


(1) anas kanc̉a $<$ aña-š kanča
(2) hinčis pampa $<$ hinči-š pampa
(3) ċawpis $<$ cawpi-š
(4) tawlis pampa $<$ tawIi-š pampa
(5) mašra oqo $<$ mašra oqos $<$ mašra oqoš $<$ mašra oqošqa
(6) oqo pampa $<$ oqos pampa $<$ oqoš pampa $<$ oqošqa pampa

Es un cambio interno que consiste en el adelantamiento, a una posición alveolar, de la africada palatal sorda / $\breve{s} /$ que, en estos ejemplos, representa al antiguo morfema adjetivador de nombres $*$ šqa $>$ š. En los ejemplos (5) y (6), este morfema evoluciona a tal punto que termina por desaparecer. Además, en el ejemplo (1) se observa un cambio anterior en el quechua, la despalatalización de $/ \tilde{\mathrm{n}} /$, este cambio es irregular porque no afecta a todas las provincias en las que se ha completado el cambio, como si en las provincias del Callejón de Huaylas.

2. En el nivel morfológico, el proceso de hominalización verbal con el uso del sufijo derivativo: el agentivo - q, el infinitivo-y, elnominalizador-na, el participio aru -ta y el participio imperfectivo-nqa (ver cuadro 13) y la derivación nominal, con el uso del sufijo adjetivador -sh, el posesivo -yuq, el derivativo aru -ni y el sufijo locativo-pi (ver cuadro 14). Dentro del proceso de lexicalización, también se observa el procesode construcción de nombres: por sintaxis (adjetivo + sustantivo) y por composicion (sustantivo +sustantivo), cuadros 23 , 24 y cuadro 25 , respectivanente.

CUADRO 13:

NOMINALIZACIÓN VERBAL: R.V + $\{-\mathrm{Q}\}$

(1) awa- 'Tejer'.

awa - $\mathrm{q} \quad$ 'Lugar del tejedor, lugar donde se teje'.

(2) anqa- 'Salado, salar'.

anqa $-\mathrm{q} \quad$ 'El que sala, lugar donde se sala'. ${ }^{17}$

16 La composición es un proceso de formación de palabras o unidades semanticas, a partir de la unión de elementos léxicos de uso independiente en la lengua.

17 Es posible que se haya producido un cambio de significado motivado por su caracteristica geográfica porque la zona presenta piedras y restos de diseminados de cal parecidos a una zona de extracción salina. 

(3) qaya-
'Llamar'.
qaya - q
'Llamador, lugar del que llama a grandes voces".
(4)
churi - yaa ${ }^{18}$
churi - yaa $-q$
'Volverse infértil, improductivo'.
(5) luya-
'Lugar de tierra infértil'. 'Tierra estéril'
- Blanco'. Probablemente esta raiz indique una acción o estado. además de su función adjetival.
luya -q luya $-q \cdot E l$ que blanquea', 'Cerro que blanquea a la zona por el esparci- miento de cal".

El morfema nominalizador de verbos $\{-\mathrm{q}\}$, unido a una raiz verbal, asigna una función agentiva a los topónimos formados por raíces verbales, es decir, estos nombres van a designar el lugar del que ejecuta la acción mencionada por la raiz o el lugar que ejecuta la acción. En el ejemplo (4), se observa la presencia de dos sufijos, un nominalizador de verbos -yaa y el otro, un derivador de nombres - q. En este caso, la raíz nominal 'churi' se convierte en un verbo intransitivo que denota lo que refiere la raiz. Así, churi-yaa significaria 'volverse varón' que ubicándolo en el contexto geográfico seria 'volverse estéril'. Esta raiz está acompañada por el sufijo agentivo -q, nominaliza el tema verbal, asignando una función nominal a la raiz.

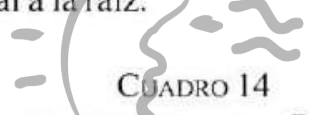

NOMINALIZACION VERBAL: R.V $+\{-Y\}$

(1) Purwa-y 'Juntar'.

Purway punta Bicumbretondesedeun efganade. (THQC)

(2) Mismi- "Jjiltgarse (etagna)i hemejarsed(tatiorra); extenderse (el fuego o incendio).

Mismi- y 'Lugar de filtraciones', 'Quebrada adecuado para hacer fuego'.

El infinitivo $\{-y\}$ morfema de nominalización verbal aparece siempre unido a una raiz verbal. La acción verbal que expresa es abstracta, porque no indica ni tiempo ni aspecto, derivando siempre en sustantivos abstractos.

\section{CUADro 15}

NOMINALIZACIÓN VERBAL: R.V $+\{-n a\}$

(1) qompay-

qompay-na
Tirar, arrojar'.

'Lugar de tiro, de pase de objetos de una orilla a la otra'.

18 Parker lo llama sufijo transformativo. En Parker, 1976: pp. 138,139. 


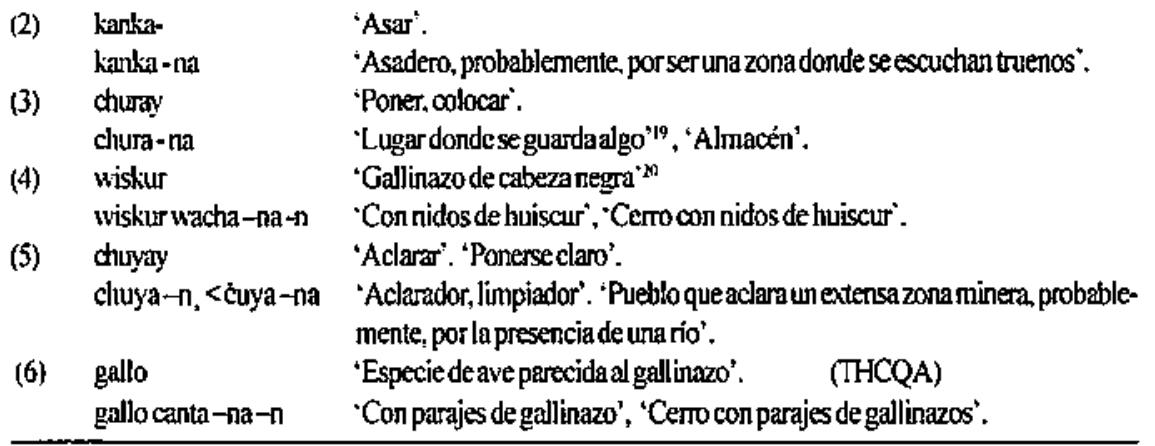

En la mayoría de los casos el concretizador -na nominaliza la raíz verbal, asignándole al topónimo que lleva este sufijo una idea más concreta e indica un lugar caracterizado por la acción que expresa la raíz verbal. En (4) el morfemana adquiere la función de participio potencial, indicando la realización futura de una acción. En el caso (5) la vocal del sufijo cayó en final de palabra por el proceso de debilitamiento que sufren en algunos casos las vocales, el sufijo parece haberse lexicalizado, dando referencia locativa.

- Cuadro 16

NOMINALIZACION VERBAL: R.V $+\{-$ ta $\}$

(1) Hoya $-y-$ Hoye: -ta punta

(2) QaraQara -ta pampa
"Hacer hoyas", 'Formarse hoyas'. (THCQ A)

Cumbre de hoyadas.

J'Piel corteza'-Pèlar'. dTHQAb,

'Llanura pelada, pobre (sin vegetación, ni pasto)"

El sufijo aru -ta funciona como un nominalizador de verbos, adherido a estas raíces indica una acción o estado en sentido abstracto, se puede identificar como un sufijo participial en la frase verbal, denotando la acción como un hecho realizado. Hasta ahora, sólo acompaña al elemento modificativo de la frase nominal. En el caso (2), aparece con una raiz ambivalente que puede actuar como tema nominal o tema verbal, su función estará determinada por el sufijo; mientras que, en (1) observamos una raíz derivada de procedencia castellana, acompañada de este sufijo denota una acción concluida.

19 Registrado así, por Fray Domingo de Santo Tomás, en el Lexicón o Vocabulario de la lengua general del Perú.1560.

${ }^{20}$ En Parker y Chávez. Limn. 1976. 
Cuadro 17

NOMINALIZACIÓN VERBAL: RV + -nqa:

(1) krus mata -nqa 'Cruz donde cazan animales' (THCQ).

El sufijo -nqa unido a una raíz verbal indica una acción posible o que ya ha empezado. En el siguiente ejemplo, el sufijo acompaña al elemento nuclear de la frase nominal, un tema verbal castellano.

CUADRO 18

DERIVACION NOMINAL: R.N $+\{$-yoq $\}$

(1) Qenwa- 'Variedad de árbol'

Qenwa -yoq 'Ccon quenua', 'Lugar que tiene quenua'.

(2) Onqa- De origen dudoso. Probablemente, se refiere a una variedad de planta'.

Onqa-yo ruri 'Quebrada con onca'.

El sufijo derivador de nombres yog, se añade a una raiz nominal para señalar al posesor de lo que expresa la raiz, este sufijo aparece ya fusionado, proveniente de un verbo desaparecido y un sufijo marcador de agente, porque refiere una oración 'lugar o ente que tiene algo'. El derivativo yuq aparece inmediatamente después del elemento nuclear, en el ejemplo (2) acompaña al elemento modificativo de la frasenominal. Siempreza a caracterizar el lugar con la presencia de algo (vegetal o animald 1i Converso "

\section{CUADro 19}

DERIVACIÓN NOMINAL: R.N + sh

(1) chawpi

chawpi $-\mathrm{S}$

(2) hinchi

hinchi -s pampa

(3)

'Centro, punto medio'.

'Con punto medio', 'Quebrada ubicada entre dos comunidades'.

'Fuerte'.

'Llanura con superficies duras, macizas'.

'Especie de planta espinosa'.

"Con jeco o jecosh, "Cerro de jecosh".

Por la descripción geográfica y la estructura del topónimo puede estar relacionado con el verbo hiqay 'caerse', pero es necesario hacer un estudio detallado posteriormente. 

(4) kuru $>$ ouru 'Gusano'21. ichik øuru -sh 'Con pequeños gusanos', 'Laguna con pequeños gusanos'.
(5) llama 'Ganado camélido'.
(6) uru 'Pequeño'.
uru -sh qucha 'Laguna empequeñecida'.
(7) qarwa 'Verde amarillento'.
qarwa -sh ruri 'Quebrada con pastos maduros'.
(8) tawlli- 'Tipo de legumbre, muy parecido al chocho'.
tawlli -sh kancha 'Estancia con taulli, donde abunda el taulli'.

El morfema derivativo -sh puede aparecer en la formación de temas verbales y nominales. En la toponimia de Ticapampa es frecuente la presencia del sufijo -sh en raíces nominales, designando diferentes especies de vegetales. Añadido a una raiz nominal funciona como un elemento adjetivador dentro de la frase, caracterizando el lugar por la presencia particular de algo. Mayormente, acompaña al elemento modificativo de la frase nominal. Este morfema proviene de la partícula *-šqa > -ša > -š > s > $\varnothing$; que termina por desaparecer en algunos casos.

Se puede decir que las formas (1) y (2) presentan la forma castellanizada del morfema, el resto de los ejemplos poseen la forma quechua.

\section{CUAARO 20}
(1) pinkacha
(2) chipta
(3) tumshu
(4) poqra $-q$

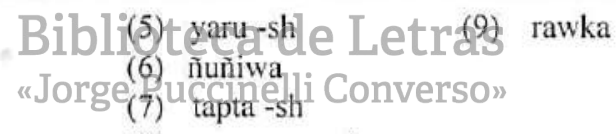
(8) onga -yo ruri

Según la información recogida, a través, de los informantes, los ejemplos (2), (3), (5) y (7) aluden a tipos de plantas silvestres propias del lugar, algunas conocidas por sus propiedades curativas; el ejemplo (7) hace referencia al nombre común de una ave típica de la zona..

\section{CUADRO 21}

DERIVACIÓN NOMINAL: R.N + $\{-$ pi $\}$
(1) ukru- 'Hoyo'.
ukru -pi 'En los hoyos', 'Llanura de hoyos', 'Lugar de hondonadas'.

\footnotetext{
${ }^{21}$ Significado recogido en Vocabulario poliglota incaico y en Diccionario Ancash Huaylas de Parker y Chávez.
} 
El sufijo de flexión de caso, el locativo-pi $\mathrm{i}^{22}$, presente en el PQ y frecuente en las variedades del QII, aparece en la variedad quechua Ancash Huaylas que posee un sufijo de igual función:-chaw. Generalmente, los sufijos de caso tienen la función de marcar la relación que existe entre el sujeto y el verbo, especificamente este sufijo da referencia de lugar.

\section{CUADRO 22}

DERIVACIÓN NOMINAL: R.N $+\{-n i\}$

(1) isku pachaisku pacha $-n$

(2) kondor wa yikondor wayi $-n$

(3) kishwar pachakishwar pacha $-n$

(4) pacha- posada 'Posada, descanso de pendiente'. (THQA C) pacha $-\mathrm{n}$ posada

(5) rikana-hirk rikana-n hirka

(6) wachaw- tsaka wacho $-\mathrm{n}$ tsaka

(7) pishqo wayi pishqo wayi $-n$
'Pendiente de cal'. (THQA)

'Con pendientes de cal', 'Cerro que tiene pendientes de cal'.

'Casa del cóndor'. (THQA)

'Con parajes de cóndor', 'Cerro con parajes de cóndor'.

'Pendiente del quishuar (especie de planta)". (THQA)

'Con pendientes de quishuar', 'Cerro con pendientes de quishuar'.

'Descanso con pendiente'.

'Cerro minador, cerro donde se ven las estrellas'. (THQA)

'Cerrocon mirador'.

'Puente de huachóu (especie de planta)'. (THQA)

'Puente que tiene huachón o hecho de huachón'.

'Casa del pishco'. (THQA)

Considerando que parto del área geográfica de la variedad QI fue alcanzada por la expansión de la familia lingüistica aru, según investigaciones lingüistico-históricas ${ }^{23}$, postulamos la existencia del derivativo -ni, como un morfema que evidencia el contacto quechua aru, observable en la toponimia de Ticapampa. Por otro lado, la variedad QI posee un sufijo de flexión nominal $\{-n\}$ marcador de tercera persona, que se agrega a la raiz cuando acaba en vocal corta. Pero este morfema de flexión no corresponde a un proceso de derivación nominal.

El sufijo aru -ni, marca el sujeto poseedor de lo que denota la raiz nominal, siempre va a aparecer después de la raíz nominal a la cual deriva. Este sufijo

22 Parker refiere a la presencia de este sufijo como modismos, adaptados en esta variedad, producto de temas derivados de manera irregular. En Parker, 1976, pp.84.

${ }^{23}$ Según el mapa de las lenguas andinas a principios del s. IX, en el que la familia linguística aru ocupó parte la Costa, el Centro y el Sur del Perú. Cerrón Palomino y otros, 1983.

${ }^{24}$ En los casos 5) y 10) los sustantivos ocupan la posición de elementos adjetivados, modificando el núcleo que es el adverbio 'ruri', cambiando función éste; en el caso 10), se observa el uso de nombres patronímicos vinculados con el lugar por relaciones de pertenencia o posesión. 
puede aparecer acompañando a un elemento nuclear o a un elemento modificativo. Morfológicamente es más o menos equivalente al derivativo quechua yoq, aparece acompañando al elemento nuclear de la frase nominal y, en otros casos, al elemento modificativo. La posición final de palabra (en posición postónica) obliga a la caída de la vocal del morfema, éste aparece en todos los casos sin vocal.

\section{Cuadro 23}

\section{Proceso de Lexicalización}

CONSTRUCCIÓN POR SINTAXIS
(1) Ismu Cancha
(7) Puca Rajo Punta
(2) Ichic Marca
(8) Ichic Huishca
(3) Hatun Pampa
(9) Ichic Urush
(4) Hatun Huishca
(10) Hatun Pucru
(5) Quenua Ruri (THQC)
(10) Torre Ruri
(THCQ)

Estos compuestos se puede observar ła lexicalización como un proceso que ocurre en el nivel morfosintáctico, se inicia con la unión de unidades morfofuncionales a una base léxica para la formación de una unidad léxico-semántica. En la toponimia de Ticapampa, se observa la formación de nombres, a partir de la unión de unidades independientes, en las que una de ellas es un adjetivo y tiene la función de modificar el núcleo de la frase; el núcleo lo constituye un sustantivo, que ocupa siemprelalposición final del comípuesto, (a ecepción de los casos) (5) y (10). Genejalmente, se les reconoce porque las unidades de los compuestos no se encuentran gráficamente unidas. La frase nominal está constituida por:

$$
\begin{aligned}
\mathrm{FN}= & \{\text { F. ADJ }\{\text { Adj }\}+\text { N. Frasal }\{\text { Sust. }\} \\
& \{\text { F. ATrib. }\{\text { Sust. }+ \text { Sus. }\}+\text { N. Frasal }\{\text { Sust. }\}
\end{aligned}
$$

El orden de los elementos constituyentes es rígido de acuerdo a la tipología SOV del quechua.

\section{CUADro 24}
(1) Monte Bello
(5) Alto Perú
(2) Buenos Aires
(6) San Lorenzo
(3) Planta Nueva
(7) Carmen Alto
(4) Santo Domingo de Compina
(8) Santo Domingo de Jinchis 
En este cuadro observamos otro caso de formación de nombres, en que los compuestos no se llegan a unir gráficamente, pero sí léxico-semánticamente. Bajo este proceso observamos la formación de frases con una estructura:

F.N: $\quad$ Modificador + Núcleo $\}$

\{Núcleo + Modificador\}

Los ejemplos (4), (6) y (8) constituyen nombres de personajes religiosos llegados con la Conquista, en otros casos, muestran una construcción nominal peculiar del periodo Colonial, caracterizada por anteceder al nombre o núcleo el adjetivo 'santo'; connotando los nombres de personajes y lugares importantes.

Cuadro 25

CONSTRUCCION POR COMPOSICIÖN

(1) Huancapeti

(2) Tomapata (THCQ)

(3) Querococha

(4) Patococha (THCQ)

(5) Ticapampa

La interpretación más cercana de estos compuestos sería:

(1) Cubierto de rocas; 7 Cumbre de rocas'

(2) Andén de descansos, Andên cơn descansos.

(3) 'Laguna de árboles' (raguna lionde se observarestos dé árboles).

(4) 'Laguna de patos silvestres'

(5) 'Llanura de adobe' 24

(6) 'Cueva de forma de cerdo'

(7) 'Manantial de oro'

(8) 'Cerro de metales preciosos'

(9) 'Llanura de canlla'

(10) 'Puente de piedra'

La composición es un proceso de formación de palabras que consiste en la unión de una unidad léxica independiente (sustantivo) con otra unidad para formar una sola unidad morfofuncional y semántica. Los elementos que comprenden la frase son de carácter nominal, donde el elemento principal es el

${ }^{25}$ En Parker y Chávez, 1976. 
elemento denominador de la frase y el elemento codificador, lo constituyen los elementos especificadores del núcleo. En los ejemplos presentados se produce una fusión léxica y gramatical del compuesto, éste designa un ente geográfico, con una estructura gramatical constituida por un elemento modificador del núcleo y por un núcleo, que ocupa siempre la posición final en los compuestos, ambos elementos son sustantivos.

Los topónimos como nombres de espacios geográficos hacen referencia a un ente geográfico en particular, esta función hace posible que podamos decir que el topónimo es un nombre propio, que distingue a una entidad geográfica de otra, dentro de un espacio geográfico común.

Las palabras compuestas pierden el acento del primer elemento, conservando sólo el del segundo elemento o núcleo. Ejemplo:

Ticapampa [tika pâmpa] 'Llanura donde se hacian adobes'.

La frase nominal puede estar constituida por un solo elemento (palabra simple) o por más de dos, pero sólo uno de ellos es el núcleo o elemento principal de la frase, éste se ubica siempre al final. El núcleo es siempre un sustantivo y los modificadores más frecuentes son otros sustantivos, según Parker, funcionan como sustantivos atributivos del núcleo; también encontramos, como modificadores, frases adjetivas. . Para los procesos de construcción de palabras en español, Manuel Alvar habla aeerea de una relaeión de identidad, establecida entre los elementos de útebripuesto-lexical, quel- loshace pertenecer a una sola clase por naturaleza. Ahora, tomando en cuenta la tipología de la lengua quechua, el lugar del elemento denominador o nuclear será al final, por ejemplo:

$$
\begin{gathered}
\text { Ichu marka < ichu "pasto duro", marka } \\
\text { "zona, pueblo, área" } \\
\text { "zona, área que es de ichu" } \\
\text { "zona, área de abundante ichu" }
\end{gathered}
$$

Se han encontrado topónimos construidos por reduplicación de las raíces nominales, la reduplicación parece dar énfasis a la frase nominal, relacionándola con alguna característica particular del lugar, como marcar el rasgo de cantidad, de algo referido a un tipo de vegetal o mineral, porque, estos nombres van a nominar extensas áreas, como cerros, llanuras y nevados. 
(1) Puru

Puru Puru

Puru Puru Punta

(2) Puca

Puca Puca

(3) Luyac

Luyac Luyac ${ }^{26}$
'Tipo de recipiente pequeño (de calabaza) ${ }^{23}$.

'Granadilla'.

'Cerro donde abunda el puru'.

'Rojo, colorado'.

'Lugar (cerro) enrojecido'.

'Blanco, el que blanquea'.

'Lugar (cerro) blanco, lugar que blanquea'.

4. De acuerdo al corpus toponímico, la lengua de mayor influencia es el quechua con el $76 \%$ del total de nombres, le sigue el castellano con $13 \%$ de topónimos híbridos castellano-quechua y el aru, con los híbridos quechuaaru. A través de este trabajo se prueba el contacto de lenguas distintas: por un lado, la lengua quechua que ya estaba extendida y; por el otro, las lenguas aru que se expandieron por esas zonas. Algunos topónimos son oscuros semánticamente, debido a que su origen es desconocido, en otros casos, los elementos que caracterizaban a diferentes entidades geográficas han desaparecido con el transcurso del tiempo, sin antes ser recogidos en vocabularios, crónicas, etc., muchos de ellos, nombres propios de plantas y animales.

5. El mecanismo más frecuente de formación es la composición dentro de él observamos la reduplicación de nombre y adjetivo (ver cuadro 26), expresando una característica de grado, cantidad, abundahcia por las extensas áreas geográficas que nominan."Jorge Puccinelli Converso»

6. En el nivel semántico, se observan las relaciones de homonimia, polisemia, alonimia e hiponimia, relaciones en las que se puede clasificar una toponimia de acuerdo a la naturaleza del ente geográfico a la que nomina.

7. La toponimia de Ticapampa contribuye como una fuente de datos históricos a los estudios topográficos de la región, además de ampliar los conocimientos culturales y lingüísticos, porque describe la extensión geográfica y los recursos naturales de un área y da información del aspecto histórico, social y etnolingüístico de un pueblo.

2s Esta palabra ha sufrido una permutación silábica (metátesis) a partir de yuraq. 
8. Los topónimos como nombres propios de entes geográficos referidos a la topografía, la zoografía y la fitografía de una región, constituyen palabras simples, derivadas y compuestas; creados por mecanismos de construcción de orden quechua, castellano o híbridos (castellano-quechua, quechua-castellano, quechua-aru). Estas palabras van a expresar o significar acciones (verbos), características de posesión (adjetivos) y nombres de entes de entes geográficos (sustantivos), como, palabras simples sin derivación.

Finalmente, podemos decir que hemos alcanzado los objetivos que nos propusimos: la recolección de topónimos del habla viva, la descripción y comprobación de los resultados, para lo cual recurrimos al método de la geografia lingüística.

\section{BIBLIOGRAFIA}

ALVAR EZQUERRA, Manuel. La formación de las palabras en español. Arco libros. Madrid, 1996.

BERTONIO LUDOVICO. Vocabulario de la lengua aimara. Juli. Puno 1612.

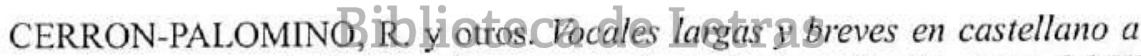
través dejor peéstamosnenlel quecturas wanka. En Actas del IV Congreso de ALFA. CILA. 1975.

- "Notas para un estudio cientifico de la toponimia quechua". En San Marcos $\mathrm{N}^{\circ} 17$, pp. 189-211. Lima, UNMSM, 1976.

Lingüística Quechua. Centro de Estudios Rurales Andinos "Bartolomé de Las Casas". Cuzco 1987.

El Diccionario quechua de los académicos: cuestiones lexicográficas, normativas y etimológicas. En Revista Andina $\mathrm{N}^{\circ} 1$, pp.151-206. Cuzco. Centro de Estudios Regionales Andinos "Bartolomé de Las Casas", 1997.

Guia para estudios de toponimia. CILA. UNMSM. 1976.

CONCEJO DISTRITAL DE TICAPAMPA. Realidad socioeconómico y cultural de Ticapampa. Provincia Recuay, Región Chavin. 1996. 
GONZÁlez HOLGUÍN, Diego. Vocabulario de la lengua de todo el Perí, Ilamado lengua Quechua o del Inca. Edición facsimilar de la versión 1952. Editorial UNMSM. 1989.

INSTITUTO GEOGRÄFICONACIONAL.Carta Nacional de Recuacy a 200,000. 1934.

- Carta Nacional de Recuay a 100,000. Hoja 20. 1970.

- Directorio Nacional de Centros Poblados. 1993.

- Mapa distrital de Ticapampa. Escala de 25000. INEI 1993.

MASGO CABELLO, H. y BALDOCEDA E., Ana. Etimologia de topónimos de Huaros, Lima, INVEL. UNMSM. Informe final de un proyecto anual de investigación. 1996.

OFICINA CENTRAL DE CATASTRO RURAL.Carta Nacional de Ancash a 25000, hojas 20:-III N, 20i-IV- SO. 1973.

PACKER, Gary y CHÁVEZ, Amancio. La clasificación genética de los dialectos quechuas. En Revista del museo Nacional. Tomo XXXII. Lima. 1976.

- Gramática quechua: Ancash-Fnailas. Ministerio de Educación. Instituto de Estudios Peruanos. Lima. Perú. 1976.

Diccionario Ancash-Huailas. Ministerio de Educación. Instituto de Estudios Peruanos. Lima.

RAIMONDI, Antonio. Et departamento de Ancash, Sus riquezas minerales.

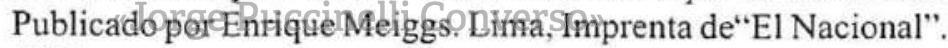
1873.

- Mapa del departamento de Ancash, con la nueva provincia Dos de Mayo del departamento de Huánuco, a escala de 100 km. (2 milimetros por kilómetro), 1876.

SANTO Tomás, FRAY Domingo, Gramática o arte de la lengua general de los indios de los reynos del Perí. Estudio introductorio y notas por Rodolfo Cerrón-Palomino. Centro de Estudios Regionales Andinos "Bartolomé de Las Casas". Cuzco. (1560) 1995.

- "Lexicón, o Vocabulario de la lengua del Perí" UNMSM. Publicación del Cuarto Centenario. 1951.

SOLIS FONSECA, Gustavo. La gente pasa, los nombres quedan... Ediciones Lengua y Sociedad. Lima. 1997. 